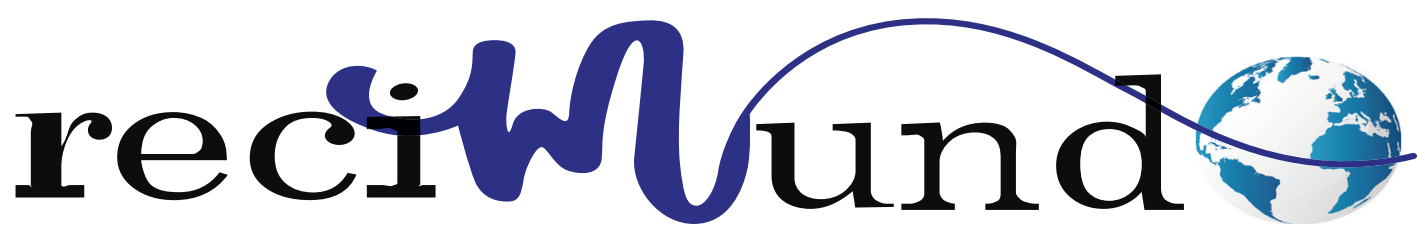

Revista Científica Mundo de la Investigación y el Conocimiento

DOI: 10.26820/recimundo/5.(1).enero.2021.191-206

URL: http://recimundo.com/index.php/es/article/view/998

EDITORIAL: Saberes del Conocimiento

REVISTA: RECIMUNDO

ISSN: 2588-073X

TIPO DE INVESTIGACIÓN: Artículo de Revisión Código UNESCO: 32 Ciencias Médicas

PAGINAS: $191-206$

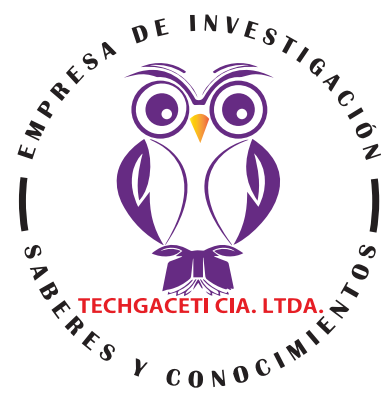

\title{
Causas de hemorragia postparto
}

Causes of postpartum hemorrhage

Causas da hemorragia pós-parto

Madeline Carolina Astudillo Córdova1; Tania Melissa Landín Ramírez; Andrea Jeanette Vélez Vera33;

Wendy Vanessa Villavicencio Briones ${ }^{4}$

\section{RECIBIDO: 28/11/2020 ACEPTADO: 06/12/2020 PUBLICADO: 31/01/2021}

1. Médico General de la Universidad de Guayaquil; Médico General Libre Ejercicio Omni Hospital; Guayaquil, Ecuador; madelineastudillo@gmail.com; (iD https://orcid.org/0000-0001-6189-0375

2. Médico General de la Universidad Católica de Santiago de Guayaquil; Médico Residente Hospital Luis Vernaza; Guayaquil, Ecuador; tlandin2@gmail.com; (iD https://orcid.org/0000-0001-6017-2398

3. Médico General de la Universidad Católica de Santiago de Guayaquil; Médico Residente en Instituto Oncológico Nacional de Sociedad de Lucha contra el Cáncer (SOLCA); Guayaquil, Ecuador, andreavelez0792@gmail.com; (DD https://orcid. org/0000-0002-6662-0603

4. Médico General de la Universidad de Guayaquil; Médico Residente Hospital Luis Vernaza; Guayaquil, Ecuador; wendyvanessa21@gmail.com; (D) https://orcid.org/0000-0001-5163-9283

CORRESPONDENCIA

Madeline Carolina Astudillo Córdova

madelineastudillo@gmail.com

Guayaquil, Ecuador

(C RECIMUNDO; Editorial Saberes del Conocimiento, 2021 


\section{RESUMEN}

La hemorragia postparto (HPP) es considerada una emergencia obstétrica y es una de las cinco principales causas de mortalidad materna a nivel mundial y la causa principal de mortalidad materna en países de ingresos bajos. La hemorragia posparto es la segunda causa más importante de muerte materna en Ecuador. Por lo anterior se plantea realizar un compendio de las causas de Hemorragias Maternas y así brindar una actualización del tema disponible para el campo médico en general, bajo la premisa de que esta complicación obstétrica no se le ha otorgado la importancia necesaria para entenderla como una real emergencia médica. Considerando las herramientas de búsqueda tecnológicas disponibles se ha de encontrar las publicaciones científicas que nos permita abordar, de manera actualizada, las causas de la Hemorragia postparto. En la revisión bibliográfica realizada hemos encontrado, principalmente que la muerte es completamente evitable si hay un diagnóstico oportuno y una estimación sincera de que las HPP son una emergencia para tratarse como tal. Por ultimo pero no menos importante, ésta complicación femenina no dispone de datos estadísticos vigentes. Un estudio señala como causas más frecuentes: la atonía uterina, los traumas/laceraciones, la retención de retos placentarios post concepción y las alteraciones de la coagulación. Algunos autores consideran a la atonía uterina como la causa más frecuente; responsable del 50 al $70 \%$ de las hemorragias post alumbramiento y del $4 \%$ de todas las muertes maternas. También, el desgarro del suelo pélvico es una causa a tomar en consideración, ya que el mismo va acompañado de una denervación de la musculatura del suelo pélvico por daño en los nervios pudendos y sacros, a consecuencia de partos vaginales, la cual pudo deberse a la instrumentación empleada en la atención del parto.

Palabras clave: Hemorragia Postparto, Emergencia Obstétrica, Mortalidad Materna.

\section{ABSTRACT}

Postpartum hemorrhage (PPH) is considered an obstetric emergency and is one of the top five causes of maternal mortality worldwide and the leading cause of maternal mortality in low-income countries. Postpartum hemorrhage is the second leading cause of maternal death in Ecuador. Therefore, it is proposed to make a compendium of the causes of Maternal Hemorrhages and thus provide an update on the subject available for the medical field in general, under the premise that this obstetric complication has not been given the necessary importance to understand it as a real one. medical emergency. Considering the available technological search tools, it is necessary to find the scientific publications that will allow us to address, in an updated way, the causes of Postpartum Hemorrhage. In the bibliographic review of death we have found, mainly that death is completely avoidable if there is a timely diagnosis and an estimate since PPH is an emergency to be treated as such. Last but not least, this female complication does not have current statistical data. A study indicates the most frequent causes: uterine atony, trauma / laceration, retention of post-conception placental challenges and coagulation disorders. Some authors consider uterine atony as the most frequent cause; responsible for 50 to $70 \%$ of postpartum hemorrhages and $4 \%$ of all maternal deaths. Also, the tear of the pelvic floor is a cause to take into consideration, since it is accompanied by a denervation of the pelvic floor muscles due to damage to the pudendal and sacral nerves, as a result of vaginal births, which could be due to the instrumentation used in delivery care.

Keywords: Postpartum Hemorrhage, Obstetric Emergency, Maternal Mortality.

\section{RESUMO}

A hemorragia pós-parto (HPP) é considerada uma emergência obstétrica e é uma das cinco principais causas de mortalidade materna no mundo e a principal causa de mortalidade materna em países de baixa renda. A hemorragia pós-parto é a segunda principal causa de morte materna no Equador. Portanto, propõe-se fazer um compêndio das causas de Hemorragia Materna e assim fornecer uma atualização sobre o assunto disponível para o campo médico em geral, sob a premissa de que esta complicação obstétrica não foi dada a importância necessária para entendê-la como uma emergência médica real. Considerando as ferramentas de busca tecnológica disponíveis, é necessário encontrar as publicações científicas que nos permitirão abordar, de forma atualizada, as causas da Hemorragia Pós-parto. Na revisão bibliográfica da morte descobrimos, principalmente que a morte é completamente evitável se houver um diagnóstico oportuno e uma estimativa, já que a HPP é uma emergência a ser tratada como tal. Por último, mas não menos importante, esta complicação feminina não tem dados estatísticos atuais. Um estudo indica as causas mais freqüentes: atonia uterina, trauma / laceração, retenção de desafios placentários pós-concepção e distúrbios de coagulação. Alguns autores consideram a atonia uterina como a causa mais freqüente; responsável por 50 a 70\% das hemorragias pós-parto e 4\% de todas as mortes maternas. Além disso, a laceração do assoalho pélvico é uma causa a ser levada em consideração, pois é acompanhada por uma denervação dos músculos do assoalho pélvico devido a danos aos nervos pudendo e sacro, como resultado de partos vaginais, o que poderia ser devido à instrumentação utilizada nos cuidados com o parto.

Palavras-chave: Hemorragia pós-parto, emergência obstétrica, mortalidade materna. 


\section{Introducción}

La hemorragia postparto (HPP) es considerada una emergencia obstétrica, es una de las cinco principales causas de mortalidad materna a nivel mundial y la causa principal de mortalidad materna en países de ingresos bajos. La mayoría de las muertes provocadas por HPP ocurren durante las primeras 24 horas después del parto: la mayoría de estas podrían evitarse a través del uso profiláctico de agentes uterotónicos durante el alumbramiento y mediante un tratamiento oportuno y apropiado. Para prevenir la mortalidad, es importante reconocer el tiempo transcurrido desde el inicio de la hemorragia y sus causas para llevar a cabo el tratamiento adecuado. Mejorar la atención de la salud de las mujeres durante el parto para prevenir y tratar la HPP representa un paso necesario para el cumplimiento de los Objetivos de Desarrollo del Milenio planteados por la Organización Mundial de la Salud (OMS). (Vargas Chaves, 2020) (OMS, 2014) Abou-Zahr C en 2003 asegura que al rededor del $11 \%$ de las mujeres que dan a luz un nacido vivo sufren hemorragia postparto severa En América Latina, el 8.2\% de las hemorragias obstétricas que requieren transfusión ocurren en el periodo postparto, representando el $64 \%$ de las necesidades totales de transfusión, de acuerdo a un análisis realizado en 712.081 historias clínicas perinatales disponibles en la base regional del Sistema Informático Perinatal de la Organización Panamericana de la Salud, correspondientes al período 2009-2012. La letalidad por hemorragia en el período posparto estimada sobre casos válidos fue de $2.2 \%$. En mayor detalle, el $26.4 \%$ de estas gestantes presentaron hemorragias vinculadas al posparto. Las hemorragias más graves, se agruparon en el período posparto $(8.2 \%)$. (OPS, 2012)

Se plantea realizar un compendio de las causas de Hemorragias Maternas y así brindar una actualización del tema disponible para el campo médico en general, bajo la premisa de que esta complicación obstétrica no se le ha otorgado la importancia necesaria para entenderla como una real emergencia medica.

\section{Metodología}

Considerando las herramientas de búsqueda tecnológicas disponibles se ha utilizado el motores de búsqueda académicos y oficiales para encontrar las publicaciones científicas que nos permita abordar, de manera actualizada, las causas de la Hemorragia postparto.

En medio de la investigación se hace evidente la edición oficial de organizaciones internacionales para la salud, de las cueles, luego se la lectura y clasificación de cada publicación encontrada se recopila y expone la información más relevante y concreta para expresar un analítico resumen para los fines académicos planteados.

\section{Resultados}

La hemorragia posterior al parto ha sido definida, de acuerdo a las guías gineco obstétricas de la Organización Mundial de la Salud, como el desarrollo de un proceso de pérdida sanguínea, desarrollada a través del tracto vaginal de la paciente, e indistintamente si es durante el momento del parto $u$ horas después del mismo. Todo este proceso patológico requiere, para ser diagnosticado, desarrollarse en las primeras 2 a 3 horas siguientes al parto. Luego de la expulsión de la placenta, las contracciones ayudan a comprimir los vasos sangrantes de la zona donde estaba adherida la placenta. La pérdida de sangre promedio luego de un parto simple por vía vaginal es de aproximadamente $500 \mathrm{ml}$ (o alrededor de la mitad de un cuarto de galón). La pérdida de sangre promedio luego de un parto por cesárea es de aproximadamente $1000 \mathrm{ml}$ (o un cuarto de galón). (Gallegos Ojeda, 2019) 
Tabla 1. Ejemplos de definiciones de hemorragia postparto

Organización Definición hemorragia postparto

\begin{tabular}{|c|c|}
\hline $\begin{array}{l}\text { Organización } \\
\text { Mundial de la } \\
\text { Salud (OMS). }\end{array}$ & $\begin{array}{l}\text { - Pérdida sanguínea } \geq 500 \mathrm{ml} \text { dentro de las primeras } 24 \text { horas postparto. } \\
\text { - Hemorragia postparto severa: Perdida sanguínea } \geq 1000 \mathrm{ml} \text { dentro del } \\
\text { mismo rango de tiempo. }\end{array}$ \\
\hline $\begin{array}{l}\text { Colegio } \\
\text { Americano de } \\
\text { Obstetras y } \\
\text { Ginecólogos } \\
\text { (ACOG) }\end{array}$ & $\begin{array}{l}\text { Pérdida de sangre acumulada de } \geq 1000 \mathrm{ml} \text { o pérdida de sangre acompañada } \\
\text { de signos o síntomas de hipovolemia dentro de las } 24 \text { horas posteriores al } \\
\text { proceso de nacimiento independientemente de la vía de parto. }\end{array}$ \\
\hline $\begin{array}{l}\text { Royal College of } \\
\text { Obstetricians } \\
\text { and } \\
\text { Gynaecologists }\end{array}$ & $\begin{array}{l}\text { Hemorragia postparto menor ( } 500 \text { a a1000ml) y hemorragia postparto mayor } \\
\text { (>1000ml). Las subdivisiones de hemorragia postparto mayor incluyen } \\
\text { moderada o severa. }\end{array}$ \\
\hline $\begin{array}{l}\text { Atención de } \\
\text { Calidad } \\
\text { Materna de } \\
\text { California }\end{array}$ & $\begin{array}{l}\text { - Etapa 0: Toda mujer en trabajo de parto/parto. } \\
\text { - Etapa 1: Pérdida de sangre }>500 \mathrm{ml} \text { después del parto vaginal o }>1000 \mathrm{ml} \\
\text { después del parto por cesárea; o cambio en los signos vitales }>15 \% \text { o } \\
\text { frecuencia cardiaca } \geq 110 \text { latidos por minuto, presión arterial } \leq 85 / 45 \mathrm{mmHg} \text {, } \\
\text { saturación de } 02<95 \% \text {. } \\
\text { - Etapa 2: sangrado continuo con pérdida total de sangre }<1500 \mathrm{ml} \text {. } \\
\text { - Etapa 3: pérdida total de sangre }>1500 \mathrm{ml} \text { o }>2 \text { unidades de glóbulos rojos } \\
\text { empaquetados transfundidos; o signos vitales inestables; o sospecha de } \\
\text { coagulación intravascular diseminada. }\end{array}$ \\
\hline \multicolumn{2}{|c|}{ Fuente: American College of Obstetricians and Gynecologists ACOG. Practice Bulletin No. 183: } \\
\hline & \\
\hline
\end{tabular}

Fuente: (Vargas Chaves, 2020)

En la práctica es difícil estimar con exactitud la cantidad de sangre perdida. La estimación visual del volumen de sangre es prácticamente imprecisa y subestimada por el operador, lo que conlleva a un retraso en la implementación de procedimientos terapéuticos. Existen múltiples métodos para cuantificar el volumen de hemorragia, tales como: pesar gasas y compresas, utilización de probetas graduadas, gasas calibradas debajo de los glúteos con bolsas de plástico adjunta con marcas de medición, etc.
También se cuenta con nueva tecnología y aplicaciones desarrolladas para medir la saturación de la sangre en gasas quirúrgicas. Es primordial comenzar a cuantificar el volumen de la hemorragia una vez producido el nacimiento del neonato, para evitar incluir en la medición el líquido amniótico, orina y antisépticos. (Gallegos Ojeda, 2019) 


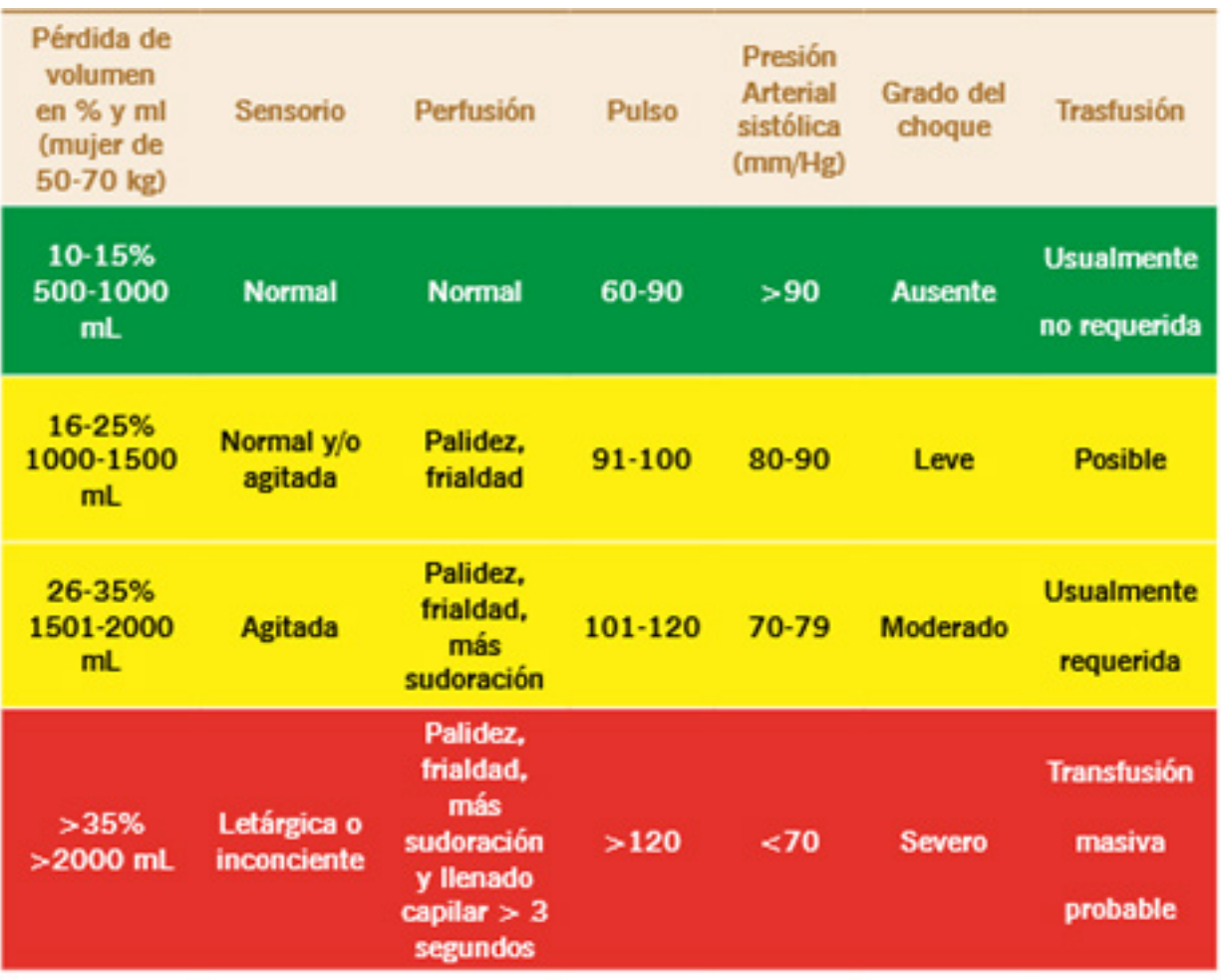

Figura 1. Diagnóstico y clasificación del grado de choque hipovolémico

Fuente: (Organizacion Panamericana de la Salud, 2019)

Los principales resultados adversos están condicionados por una estimación inadecuada en la pérdida de sangre, con una tendencia generalizada a la infraestimación. El equipo obstétrico es fundamental en la prevención de eventos adversos en la atención al parto y postparto. La comunicación eficaz dentro del equipo y el factor humano (liderazgo, toma de decisiones, capacidad para pedir ayuda, conciencia de gravedad de la situación, etc.), son determinantes en la aplicación de las intervenciones invasivas adeucadas. La coordinación del personal y la actuación rápida y eficaz de todos los profesionales, es vital para la supervivencia materna en las urgencias obstétricas. (López Martínez, 2017)

Tabla 2. Factores de riesgo para HPP

\begin{tabular}{ll}
\hline \multicolumn{1}{c}{ Factor de riesgo } & $\begin{array}{l}\text { OR ajustado } \\
\text { (análisis multivariado) }\end{array}$ \\
\hline Placenta previa & 13,1 \\
Abruptio placentae & 12,6 \\
Cesárea de emergencia & 3,6 \\
Enfermedad Von Willebrand & 3,3 \\
Corioamnionitis & 2,5 \\
Cesárea electiva & 2,5 \\
Embarazo gemelar & 2,3
\end{tabular}

Peso fetal mayor de 4500 gramos 1,9 


\begin{tabular}{ll} 
Polihidramnios & 1,9 \\
Multiparidad & 1,9 \\
Síndrome HELLP & 1,9 \\
\hline Parto instrumentado fórceps & 1,9 \\
\hline Inducción del trabajo de parto & 1,6 \\
\hline Obesidad & 1,6 \\
Antecedente de HPP & 1,6 \\
Cesárea previa & 1,5 \\
Trabajo de parto prolongado & 1,1 \\
\hline Edad mayor de 40 años & 1,4 \\
\hline
\end{tabular}

Fuente: (Organizacion Panamericana de la Salud, 2019)

La hemorragia posparto se clasifica como temprana o tardía. La hemorragia temprana es la que se presenta durante las primeras 24 horas del periodo posparto generalmente en las 2 primeras horas siendo la más frecuente y grave; las causas son atonía uterina, retención de restos placentarios, anormalidades placentarias y laceraciones del tracto genital. La hemorragia tardía es la que ocurre entre las 24 horas y las 6 semanas del posparto, con una frecuencia entre el 5 y $10 \%$ de los partos, las causas más comunes son retención de restos placentarios, infecciones, laceraciones y la enfermedad trofoblástica. (Organizacion Panamericana de la Salud, 2019)

Alvaro et al (2016) establecen que las causas de la Hemorragia Postparto (HPP) Primaria se consideran una alteración de uno o más de 4 procesos, que corresponden a la regla nemotécnica de "las cuatro T".

TONO: Anatomia uterina. Es la causa mas frecuente de HPP y provoca un $75-90 \%$ casos de la misma. Puede aparecer en cualquier via de finalización de la gestación:

- Cesarea: la incidencia en este caso puede llegar a alcanzar hasta el 6\%.

- Parto vaginal: existen menos estudios que para el riesgo de atonía en cesárea.
TRAUMA: Por lesión uterina, cervical o vaginal $10-20 \%$

- Desgarros de tercer y cuarto grado perineal.

- Ruptura de ligamentos

- Desgarros cervicales, uterinos y vaginales

- Inversión uterina.

TEJIDO: Retencion del tejido placentario. $10 \%$. Se produce en el $0,5-3 \%$ de los partos. La mayoría de las placentas retenidas pueden ser extraídas de forma manual, pero en un porcentaje pequeñode los casos $(0,001.0,05 \%$ del total de los partos) la causa puede ser una placenta ácreta, pércreta o íncreta. Cuando se ha producido una retención placentaria en una gestante, en un futuro embarazo hay que estar alerta, ya que es una situación que tiende a recurrir.

TROMBINA: Alteraciones en la cuagulación, ya sea por una coagulopatía preexstente o adquirida. 1-3\%.

- Coagulopatías adquiridas:

- Desprendimineto de placenta

- Preeclampsia

- Sepsis

o Feto Muerto

- Embolismo de líquido amniótico

- Transfusiones de sangre incompatible 
- Coagulopatías congénitas

- Patología plaquetaria

o Trombofilias

- Enfermedad de Von Willebrand

En los casos de HPP tardía o secundaria las principales causas son:

- Subinvolución uterina

- Infección

- Retención de tejido placentario.

- Dehiscencia de la cicatriz de la cesárea.

- Anormalidad vascular, malformación Arteriovenosa.

- Coriocarcinoma.

- Hematomas (Vulvar, supravaginales)

- Coagulopatía, uso de anticoagulantes. (Alvaro A., Bejarano L., Hernández A., Iturbe, \& Ruíz C., 2016)

Sin embargo desde el punto de vista etiológico López (2017), categoriza la hemorragia posparto según la nemotecnia de las "4 t"s de la siguiente manera.

Tabla 3. Clasificación de HPP por factores de riesgo y etiología

Etiología y frecuencia

\begin{tabular}{|c|c|c|}
\hline \multirow[t]{2}{*}{ Tono $70 \%$} & $\begin{array}{l}\text { Sobredistensión Uterina } \\
\text { prolongado/precipitado } \\
\text { inducido). }\end{array}$ & $\begin{array}{l}\text { Embarazo múltiple. Macrosomia. } \\
\text { Polihidramnios. Gran Multípara. } \\
\text { Hidrocefalia severa. }\end{array}$ \\
\hline & Fatiga Muscular Uterina & $\begin{array}{l}\text { Trabajo de parto prolongado. } \\
\text { Corioamnionitis. }\end{array}$ \\
\hline \multirow[t]{4}{*}{ Trauma 20\% } & $\begin{array}{ll}\text { Desgarros } & \text { vaginales/ } \\
\text { cervicales/perineal } & \end{array}$ & Parto instrumental. Episiotomía \\
\hline & $\begin{array}{l}\text { Extensión del desgarro en la } \\
\text { cesárea. }\end{array}$ & $\begin{array}{l}\text { Mal posición fetal. Manipulación } \\
\text { brusca durante la extracción fetal }\end{array}$ \\
\hline & Ruptura uterina & Cirugía uterina previa \\
\hline & Inversión uterina & $\begin{array}{l}\text { Excesiva tracción del cordón. } \\
\text { Gran multípara. }\end{array}$ \\
\hline \multirow[t]{2}{*}{ Tejidos 9\% } & Retención de Restos & Placenta o membranas \\
\hline & Anormalidades Placentarias & $\begin{array}{l}\text { Localización: Placenta previa. } \\
\text { Invasión: acreta, percreta, } \\
\text { increta. Congénitas: Útero } \\
\text { bicórneo. Adquiridas: Cirugía } \\
\text { previa, leiomioma }\end{array}$ \\
\hline \multirow[t]{2}{*}{ Trombos 1\% } & Coagulopatías congénitas & $\begin{array}{l}\text { Hemofilia. Enfermedad Von } \\
\text { Willebrand. Hipofibrinogemia. }\end{array}$ \\
\hline & $\begin{array}{l}\text { Coagulopatías } \\
\text { embarazo }\end{array}$ & $\begin{array}{l}\text { HTA. Muerte fetal. Enfermedad } \\
\text { hepática. Síndrome HELLP. }\end{array}$ \\
\hline
\end{tabular}

Factores de riesgo 


\begin{tabular}{|c|c|}
\hline trombocitopénica & \\
\hline $\begin{array}{l}\text { Coagulación } \\
\text { diseminada }\end{array}$ & $\begin{array}{l}\text { Muerte fetal intrauterina. } \\
\text { Toxemia. Abruptio placentae } \\
\text { Embolismo amniótico } \\
\text { Sepsis }\end{array}$ \\
\hline Coagulopatía dilucional & Transfusiones masivas \\
\hline Anticoagulación & $\begin{array}{l}\text { Historia de TVP y TEP. Uso de } \\
\text { Aspirina, Heparina }\end{array}$ \\
\hline
\end{tabular}

Fuente: (López Martínez, 2017)

En el momento que se produce la detección del sangrado debe alertarse a todo el personal sanitario necesario: matrona, enfermera, obstetra y anestesista y/o médico intensivista, y al equipo quirúrgico y se activará el protocolo de hemorragia obstétrica disponible en el hospital. Cuando se ha decidido activar el protocolo de hemorragia obstétrica/hemorragia masiva, los volantes de petición de hemoderivados deben llevar una identificación, consensuada y variable para cada hospital, que ponga en conocimiento del personal de laboratorio que se trata de una situación de emergencia. Esto permite organizar y priorizar también el trabajo en el laboratorio, y evitar retrasos innecesarios. (López Martínez, 2017)

Tabla 4. Posibles intervenciones para el tratamiento de la hemorragia postparto

Intervenciones farmacológicas

\begin{tabular}{|c|c|}
\hline \multirow{2}{*}{$\begin{array}{l}\text { Droga } \\
\text { Oxitocina }\end{array}$} & Dosis \\
\hline & $\begin{array}{l}10 \text { a } 40 \text { unidades en } 500 \text { a } 1000 \mathrm{ml} \text { en solución salina infundida a una } \\
\text { velocidad suficiente para controlar la atonía o } 10 \text { unidades } \\
\text { intramusculares. }\end{array}$ \\
\hline Ácido tranexamico & $\begin{array}{l}\text { Infundir } 1 \mathrm{~g} \text { ( } 10 \mathrm{ml} \text { de una solución de } 100 \mathrm{mg} / \mathrm{ml}) \text { durante } 10 \text { a } 20 \text { minutos; } \\
\text { si el sangrado persiste después de } 30 \text { minutos, se administra una } \\
\text { segunda dosis de } 1 \mathrm{~g}\end{array}$ \\
\hline \multirow{4}{*}{$\begin{array}{l}\text { Misoprostol } \\
\text { Dinoprostol } \\
\text { Factor VIla } \\
\text { recombinant } \\
\text { humano }\end{array}$} & $\begin{array}{l}\text { Metilergonovina 0,2 mg IM cada } 2 \text { a } 4 \text { horas o ergometrina 0,5mg IV o IM } \\
\text { o ergonovina 0,25 IM o IV cada } 2 \text { horas. }\end{array}$ \\
\hline & 800 a 1000 mcg via rectal \\
\hline & 20 mg vaginal o rectal cada dos horas \\
\hline & 50 a $100 \mathrm{mcg} / \mathrm{kg}$ cada dos horas \\
\hline \multicolumn{2}{|c|}{$\begin{array}{l}\text { Intervenciones quirúrgicas } \\
\text { - Reparación de laceraciones } \\
\text { - } \text { Curetaje } \\
\text { - Sutura uterina } \\
\text { - Ligadura de arteria uterina } \\
\text { - Eigadura de arteria utero-ovárica } \\
\text { - } \text { Torniquete pélvico } \\
\text { - Escisión focal miometrial } \\
\text { - Ligadura de arteria iliaca interna }\end{array}$} \\
\hline
\end{tabular}


- Compresion arteria aorta/iliaca

- Histerectomía

Procedimientos de intervención endovasculares

- Embolización arterial selectiva

- Oclusión intermitente de arteria aorta con balón

- Oclusión intermitente de arteria iliaca común con balón

Banco de sangre

- Celulas sanguíneas empacadas

- Plaquetas

- Plasma fresco congelado

- Crioprecipitados

Intervenciones no quirúrgicas

- Masaje uterino

- Fluidos IV

- Tamponamiento uterino

Fuente: Dahlke JD, Mendoz-Figueroa H, Maggio L, Hauspurg AK, Sperling JD, Chauhan SP, Rouse DJ. Prevention and management of postpartum hemorrhage: a comparison of 4 national guidelines. Am J Obstet Gynecol

Fuente: (Vargas Chaves, 2020)

Según estudios de la Organización Mundial de la salud la reducción de la mortalidad materna en un $75 \%$ constituye el quinto Objetivo de Desarrollo del Milenio. Para alcanzar este objetivo, es preciso que los países tengan un diagnóstico exacto de las causas y los niveles de muertes maternas. En tal sentido, los esfuerzos para documentar el progreso en la reducción de la mortalidad materna deben contemplar ajustes para las inconsistencias en las cifras reportadas por los países. La aplicación de la Clasificación Estadística Internacional de Enfermedades (CIE) de la organización a las muertes ocurridas durante el embarazo, parto y puerperio CIE-Mortalidad materna (CIE - MM) se basa en la $10^{a}$ revisión de la CIE (CIE-10) y sus reglas de codificación. Busca facilitar la uniformidad de la recolección, el análisis y la interpretación de la información referente a las muertes maternas. Al mejorar la comunicación también se facilitará la codificación de las diferentes afecciones. Fundamentalmente, se pretende ayudar a los prestadores de servicios de salud, a quienes Ilenan los certificados de defunción, aclarando la aplicación de la CIE-10 y normalizando la identificación de las causas directas e in- directas de muerte. Sus principios deben ser aplicables para categorizar la información sobre las muertes recabadas a partir del registro civil, las encuestas, los sistemas de información de los hospitales, autopsias verbales, investigaciones confidenciales y otros estudios especiales. (OMS, 2012)

Dicha clasificación para la mortalidad materna por Hemorragia obstétrica se ubica en el Grupo 3 y es la siguiente:

\section{O20 Hemorragia precoz del embarazo}

Excluye: embarazo que termina en aborto (O00-O08)

O20.0 Amenaza de aborto

Hemorragia especificada como debida a la amenaza de aborto

O20.8 Otras hemorragias precoces del embarazo

O20.9 Hemorragia precoz del embarazo, sin otra especificación

O43 Trastornos de la placenta

Excluye: atención materna por crecimiento fetal deficitario debido a insuficiencia placentaria (O36.5) placenta previa (O44.-)

Desprendimiento prematuro de la

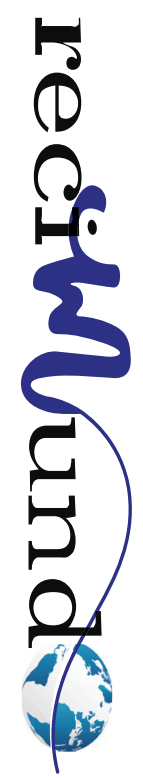


placenta [abruptio placentae] (O45.)

O43.2 Placenta ácreta (adherencia anormal)

\section{O44 Placenta previa}

044.1 Placenta previa con hemorragia Implantación baja de la placenta, SOE o con hemorragia

Placenta previa:

- marginal

- parcial

- total

SOE o con hemorragia

Excluye: trabajo de parto y parto complicado por hemorragia proveniente de los vasa previa (069.4)

045 Desprendimiento prematuro de la placenta [abruptio placentae]

O45.0 Desprendimiento prematuro de la placenta con defecto de la coagulación

Desprendimiento prematuro de la placenta con hemorragia (excesiva) asociada con:

- afibrinogenemia

- coagulación intravascular diseminada

- hiperfibrinolisis

- hipofibrinogenemia

O45.8 Otro Desprendimiento prematuro de la placenta

O45.9 Desprendimiento prematuro de la placenta, sin otra especificación

Desprendimiento prematuro de la placenta SOE

O46 Hemorragia anteparto, no clasificada en otra parte

Excluye: hemorragia en el embarazo precoz (O20.-)

hemorragia intraparto NCOP (O67.-)

placenta previa (O44.-)

Desprendimiento prematuro de la placenta normo inserta [abruptio placentae] (O45.-)

O46.0 Hemorragia preparto con defecto de la coagulación

Hemorragia (excesiva) preparto asociada con:

- afibrinogenemia

- coagulación intravascular diseminada
- hiperfibrinolisis

- hipofibrinogenemia

O46.8 Otra hemorragia preparto

O46.9 Hemorragia preparto, no especificada

067 Trabajo de parto y parto complicado por hemorragia intraparto, no clasificado en otra parte

Excluye: hemorragia preparto NCOP (O46.)

placenta previa (O44.-)

hemorragia postparto (O72.-)

Desprendimiento prematuro de la placenta [abruptio placentae] (O45.-)

O67.0 Hemorragia intraparto con defecto de la coagulación

Hemorragia intraparto (excesiva) asociada con:

- afibrinogenemia

- coagulación intravascular diseminada

- hiperfibrinolisis

- hipofibrinogenemia

O67.8 Otra hemorragia intraparto

Hemorragia excesiva intraparto

O67.9 Hemorragia intraparto, no especificada

Ruptura de útero no establecida

\section{Otro traumatismo obstétrico}

Incluye: lesión provocada por instrumentos

O71.0 Ruptura de útero antes del inicio del trabajo de parto

O71.1 Ruptura de útero durante el trabajo de parto

cuando ocurre antes del inicio del trabajo de parto

O71.3 Laceración obstétrica del cérvix

Desprendimiento anular del cuello uterino

O71.4 Laceración obstétrica vaginal alta aislada

Laceración de la pared vaginal sin mención de laceración perineal

Excluye: con laceración perineal (O70.)

O71.7 Hematoma obstétrico de pelvis

Hematoma obstétrico de:

- periné

- vagina 
- vulva (OMS, 2012)

Conscientes de esta situación y respondiendo a la estrategia de trabajo interdepartamental del nuevo Plan Estratégico 2014-2019, el Departamento de Familia, Género y Curso de Vida (FGL), la Organización Panamericana de la Salud convocó a otros departamentos a elaborar un proyecto a ser acompañado por los asesores técnicos, además de la gerencia ejecutiva de la OPS. El proyecto llamado "Cero muertes maternas por hemorragia: proyecto interdepartamental OPS" hace especial énfasis en mortalidad materna por hemorragia como intervención más sensible a la capacidad de respuesta del sistema de salud integral con un abordaje inter-programático, donde se abordarán los siguientes temas:

- aspectos preventivos (sociales, legales, culturales, económicos, médicos);

- capacidades para la atención oportuna y de calidad del parto y del postparto;

- red de servicios con mecanismos de referencia y contra referencia;

- disponibilidad permanente de sangre segura y medicamentos esenciales;

- sistemas de información y vigilancia oportuna y confiable;

- participación de la comunidad, las mujeres y los hombres. (OPS, 2012)

Sin embargo en un seguimiento realizado en 2015, De Muci expone que solo 5 países desarrollaron propuestas para participar en el proyecto y recibieron apoyo financiero directo: Bolivia, Guatemala, Haití, Perú, and República Dominicana y 5 países han desarrollado propuestas tardías y no han recibido financiación: Guyana, Honduras, México, Suriname and Paraguay (De Muci, 2015)

Aun se evidencian los esfuerzos de organizaciones mundiales abordar este tipo de emergencias con consecuencias que van más allá del resultado médico, para ello se trabaja y publica "Guías para la atención de las principales emergencias obstétricas" (2019) que pretende ser una herramienta accesible y práctica para el abordaje de las situaciones de emergencia y urgencia obstétricas más frecuentes. La razón de mortalidad materna es un indicador de las condiciones de vida y asistencia sanitaria de una población y la casi totalidad de las mismas ocurre en países en vías de desarrollo (99\%). La falta de educación, alimentación adecuada, soporte social y asistencia sanitaria se asocian fuertemente con la mortalidad materna, siendo un indicador de inequidad entre los ricos y los pobres. Así mismo, la mortalidad materna, evidencia las desigualdades de género, tanto en el acceso a la educación, la nutrición y la asistencia sanitaria. Esta situación evidencia el carácter prevenible de la muerte materna y la importancia de establecer acciones extrasectoriales que mejoren condiciones de vida e intrasectoriales que garanticen la calidad y el acceso a la asistencia sanitaria a toda la población. La muerte materna es una tragedia humana, una injusticia social y una violación del derecho a la vida. La mortalidad materna, en muchos países, se encuentra subestimada. Se debe destacar que la muerte materna, a su vez, se relaciona con graves consecuencias para la familia y la comunidad. Se asocia con abandono infantil, desnutrición, violencia y falta de desarrollo social familiar y comunitario lo que conlleva a altos costos sanitarios y sociales, siendo una grave amenaza para el desarrollo de la comunidad y el país. (Organizacion Panamericana de la Salud, 2019)

Resulta de gran importancia reconocer en forma oportuna estas complicaciones y realizar un abordaje inmediato y adecuado, de forma de evitar el agravamiento y la muerte de la mujer. El implementar acciones adecuadas, basadas en la evidencia, establecidas por protocolos de manejo y llevadas adelante por parte de personal de salud idóneo, puede descender fuertemente la razón de mortalidad materna. Analizando el impacto que sobre la MM tienen la planifi-

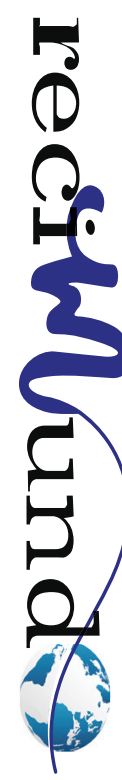


cación familiar, el control prenatal, la atención del parto por personal calificado y el tratamiento en servicios de emergencias obstétricas se encontró que el acceso a los servicios de emergencias obstétricas y a los servicios con atención segura del aborto, se asociaron significativamente con menor mortalidad materna, aún por encima de la disponibilidad de control prenatal y la buena atención del parto. Médicos, parteras profesionales o enfermeras deben tener adecuado entrenamiento en la atención del parto o embarazo no complicado y en la identificación, manejo y referencia de las complicaciones maternas. (Organizacion Panamericana de la Salud, 2019)

En esta guía propone un esquema de abordaje inicial en HPP que resulta oportuno rescatar.

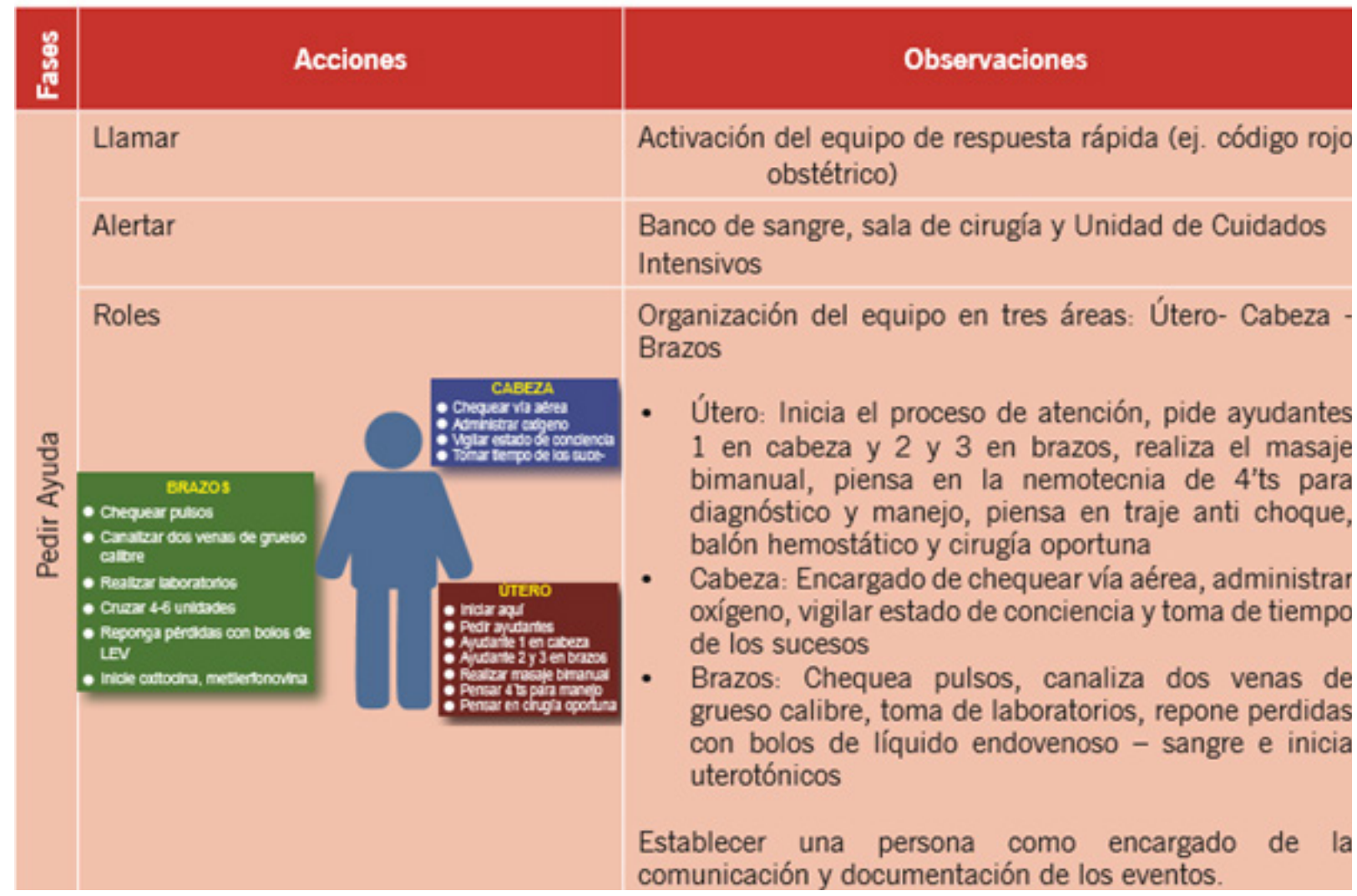

Figura 2. Esquema de abordaje inicial en HPP

Fuente: (Organizacion Panamericana de la Salud, 2019) 


\begin{tabular}{|c|c|c|}
\hline$\frac{8}{8}$ & Acciones & Observaciones \\
\hline \multirow{10}{*}{ 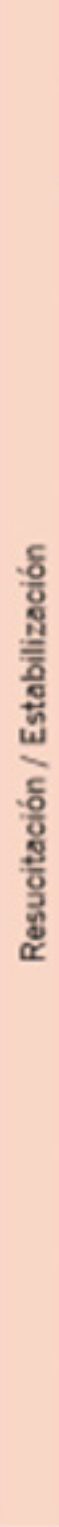 } & Vía oérea $(A)$ & Verificar vía aérea permeable. \\
\hline & Ventilación (B) & $\begin{array}{l}O_{2} \text { al } 100 \% \\
10-15 \text { litros por minuto por móscara con reservorio. }\end{array}$ \\
\hline & \multirow{8}{*}{ Circulación (C) } & $\begin{array}{l}\text { Elevación de los miembros inferiores } 30 \text { grados o posición } \\
\text { de Trendelemburg. }\end{array}$ \\
\hline & & $\begin{array}{l}\text { Colocar TAN de manera inmediata en cualquier paciente } \\
\text { con hemorragia postparto y signos de inestabilidad } \\
\text { hemodinámica (ver cuadro de TAN) (Recomendación } \\
\text { débil, evidencia de baja calidad) } 4: 15 \text {. }\end{array}$ \\
\hline & & $\begin{array}{l}\text { - Colocar } 2 \text { accesos venosos periférico percutáneos con } \\
\text { catéter calibre } 14,16018 \\
\text { - En caso de inestabilidad hemodinámica de debe } \\
\text { tener un sistema de transfusión rápido que permitan } \\
\text { calentar fluidos y productos de transfusión. }\end{array}$ \\
\hline & & $\begin{array}{l}\text { Toma de muestras: } \\
\text { - Banco de sangre: hemoclosificación, pruebos } \\
\text { cruzadas. } \\
\text { - Hemograma } \\
\text { - Pruebas de coogulación: PT, PTT, fibrinógeno. } \\
\text { - Bioquímica sanguínea: azoemia, creatinina, } \\
\text { electrolitos. }\end{array}$ \\
\hline & & $\begin{array}{l}\text { Monitoria continúa de frecuencia cardiaca, tensión } \\
\text { arterial, pulso, oximetría y gasto urinario cada hora. }\end{array}$ \\
\hline & & $\begin{array}{l}\text { Infusión de cristaloides en bolos de } 300 \text { a } 500 \propto 0 \text { (tabla } \\
\text { 6) (Recomendación fuerte, evidencia de baja calidad) } 4: 10 \text {. }\end{array}$ \\
\hline & & $\begin{array}{l}\text { Definir rápidamente la neoesidad de transfusión de } \\
\text { acuerdo a disponibilidad (tabla 6) }\end{array}$ \\
\hline & & $\begin{array}{l}\text { Aplicación de medicamentos uterotónicos y ócido } \\
\text { tranexámico (tabla 7) (Reoomendación fuerte, evidencia } \\
\text { de calidad moderada) }{ }^{43} \text {. }\end{array}$ \\
\hline \multirow{2}{*}{ 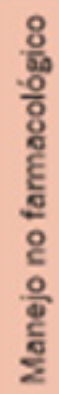 } & Masaje uterino & $\begin{array}{l}\text { Definir rápidamente si la paciente presenta una atonía } \\
\text { uterina y comenzar con masajes uterinos (Recomendación } \\
\text { fuerte, evidencia de baja calidad). }\end{array}$ \\
\hline & $\begin{array}{l}\text { Balón } \\
\text { hidrostótico }\end{array}$ & $\begin{array}{l}\text { En caso de atonía uterina la colocación de balón de Bakri o } \\
\text { condón con sonda de Foley de acuerdo a la disponibilidad } \\
\text { institucional (tabla 7) (Recomendación débil, evidencia } \\
\text { de baja calidad) }{ }^{2 a} \text { :s. }\end{array}$ \\
\hline
\end{tabular}

Figura 3. Esquema de abordaje inicial en HPP (Continuación)

Fuente: (Organizacion Panamericana de la Salud, 2019) 
Esta Guía actualizada ofrece un esquema lo mas de completo para el manejo de líquidos y hemoderivados, manejo médico y manejo quirúrgico de la HPP, además de la Evaluación de la respuesta a infusión de volumen de cristaloides $500 \mathrm{ml}$ y un Algoritmo integral del manejo médico, quirúrgico y reanimación en HPP interesante de profundizar, disponible en https://iris.paho.org/bitstream/handle/10665.2/51029/9789275320884-spa.pdf?sequence $=6 \&$ isAllowed $=y$

La información disponible en la Región de las Américas muestra grandes disparidades en la Razón de Mortalidad Materna (RMM), tanto entre países como al interior de ellos. Las mujeres que viven en condiciones de pobreza, en zonas más alejadas, con menor nivel de educación, indígenas y de otros grupos étnico/raciales y en situación de violencia de género, tienen una sobre representación en la RMM. A la vez, se reconoce que el 95\% de la mortalidad materna podría ser evitada con el conocimiento y tecnología ya disponible en la Región. La mortalidad materna tiene un alto impacto social, por ejemplo, se ha documentado que los niños que pierden la madre al nacer tienen más riesgo de morir antes de los dos años, de abandonar la escuela, sufrir desnutrición, violencia intrafamiliar, embarazos tempranos, ser víctima de trabajo infantil y prorrogar el círculo de la pobreza. (OPS, 2012) Crespo (2019) rescata datos interesantes evaluados en el Ecuador entre los que destaca el siguiente gráfico.

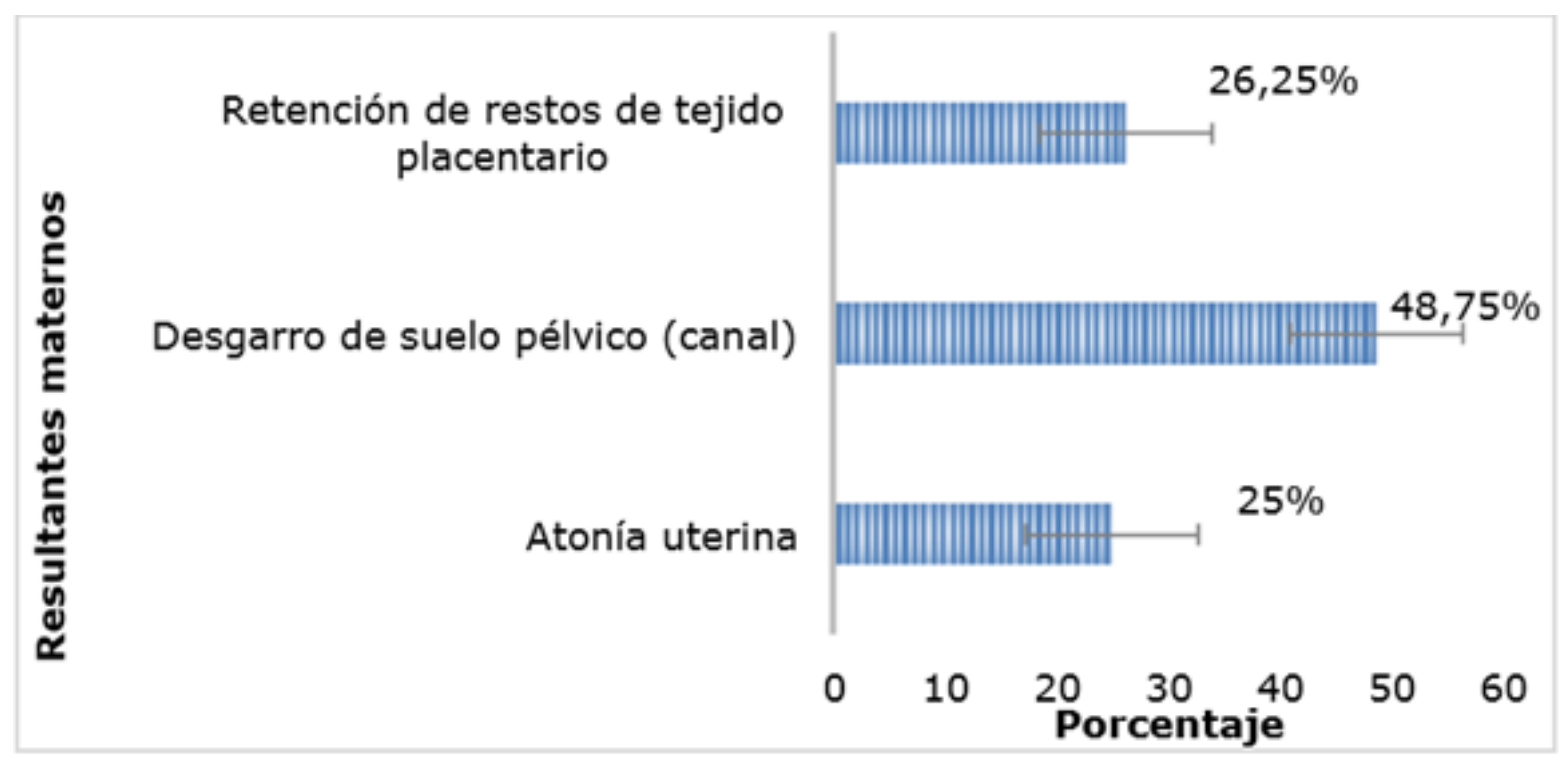

Gráfico 1. Principales causas de hemorragias postparto inmediato en el Hospital Gineco-Obstétrico Enrique C. Sotomayor, Ecuador (enero 2016-2018)

Fuente: (Crespo Antepara \& Mendieta Toledo, 2019) 
En el anterior se destaca entonces que, el desgarro de suelo pélvico suele ser la mayor causa de HPP inmediato en el centro de Salud en estudio.

\section{Conclusiones}

En la revisión bibliográfica realizada hemos encontrado, principalmente los esfuerzos de las organizaciones mundiales para sumar esfuerzos y evitar las consecuencias fatales de esta enfermedad en contra de las madres, considerando que la muerte es completamente evitable si hay un diagnóstico oportuno y una estimación sincera de que las Hemorragias Postparto son consideradas una emergencia para tratarse como tal.

Otro punto importante es que las causas son identificables y el tratamiento se va a dirigir efectiva y eficazmente siempre que se puedan identificar el origen de la hemorragia.

La hemorragia posparto, en su mayoría causada por atonía uterina, es la causa más frecuente de mortalidad materna evitable en todo el mundo, y la segunda causa más importante de muerte materna en Ecuador. (Gallegos Ojeda, 2019)

Por ultimo pero no menos importante, ésta complicación femenina no dispone de datos estadísticos vigentes, de hecho, durante nuestra investigación procuramos conseguir algún tipo de recopilación por parte de los entes oficiales y no se hizo posible. En este orden de ideas se corrobora con los comentarios encontrados dentro de los estudios abordados en Ecuador, concuerda con el seguimiento de De Munci en donde el país no figura en los esfuerzos para la reducción de muertes maternas por hemorragias y la justificación para la creación y aplicación de la CIE.

En Ecuador, aproximadamente se presentan 34.000 defunciones al año por hemorragias postparto y de acuerdo al Instituto Nacional de Estadísticas y Censos del Ecuador, en el 2015, la tasa de mortalidad materna fue de 49,2 por 100.000 nacidos vivos, siendo la hemorragia postparto una de las dos causas más frecuentes. Un estudio señala como causas más frecuentes: la atonía uterina, los traumas/laceraciones, la retención de retos placentarios post concepción y las alteraciones de la coagulación. Algunos autores consideran a la atonía uterina como la causa más frecuente; responsable del 50 al $70 \%$ de las hemorragias post alumbramiento y del $4 \%$ de todas las muertes maternas. También, el desgarro del suelo pélvico es una causa a tomar en consideración, ya que el mismo va acompañado de una denervación de la musculatura del suelo pélvico por daño en los nervios pudendos y sacros, a consecuencia de partos vaginales, la cual pudo deberse a la instrumentación empleada en la atención del parto. (Crespo Antepara \& Mendieta Toledo, 2019)

Entre 2012 y 2019 la actualización de la Guías para atención de las Principales Muertes Obstétricas nos deja como lección que la definición, causas y abordajes, al menos en cuanto a las hemorragias postparto, que es el tema en estudio, no han variado, pero por alguna razón los abordajes se han separado entre una guía y otra, mostrando con mayor simplicidad los protocolos médicos a seguir en los casos necesarios.

\section{Bibliografía}

Alvaro A., A., Bejarano L., P., Hernández A., M., Iturbe, M., \& Ruíz C., L. (2016). Manejo de la Hemorragia Postparto. Madrid: Liber Factory.

Crespo Antepara, D., \& Mendieta Toledo, L. B. (2019). Contexto de las hemorragias, en el puerperio inmediato. Memorias del Intituto de Investigación en Ciencias de la Salud, 3(17), 5-9.

De Muci, B. (2015). Cero muertes maternas por hemorragia Origen, coordinación y evolución del proyecto. Ciudad de Panamá: Organizacion Mundial Para la Salud.

Fescina R, D. M. (2012). Guías para la atención de las principales emergencias obstétricas. Monte-

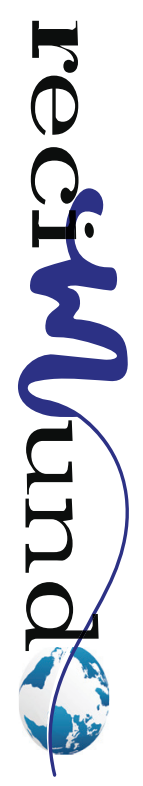


video: Publicación científica CLAP/SMR Nº 1594 Centro Latinoamericano de Perinatología Salud de la Mujer y Reproductiva CLAP/SMR.

Gallegos Ojeda, E. P. (2019). Causas de las hemorragias postparto. Guayaquil: Universidad de Guayaquil. Facultad de Ciencias Médicas. Carrera de Obstetricia.

López Martínez, C. (2017). La Hemorragia Postparto: Descripción, Manejo y Tratamiento. Santader: Escuela Universitaria de Enfermería Casa de Salud Valdecilla.

OMS. (2012). Guía de la OMS para la aplicación de la CIE-10 a las muertes ocurridas durante el embarazo, parto y puerperio: CIE-MM. Ginebra: Organización Mundial de la Salud.
OMS. (2014). Recomendaciones de la OMS para la prevención y el tratamiento de la hemorragia posparto. (E. d. OMS, Ed.) Recuperado el 26 de enero de 2021, de www.who. int: https://apps.who.int/iris/bitstream/handle/10665/141472/9789243548500_spa.pdf;jsessionid =8C8EAB49207B56688782E9AD3B6553E0? sequence $=1$

OPS. (2012). Cero muertes maternas por hemorragia: proyecto interdepartamental OPS . Recuperado el 27 de enero de 2021, de www.paho.org: https://www. paho.org/clap/index.php?option=com_content\&view=article\&id=235: cero-muertes-maternas-por-hemorragia-proyecto-interdepartamental-ops\&ltemid $=215 \&$ lang $=e s$

Organizacion Panamericana de la Salud. (2019). Guías para la atención de las principales emergencias obstétricas. Washington DC: Publicación científica CLAP/SMR N ${ }^{\circ} 1594$ Centro Latinoamericano de Perinatología Salud de la Mujer y Reproductiva CLAP/SMR.

\section{CITAR ESTE ARTICULO:}

Astudillo Córdova, M. C., Landín Ramírez, T. M., Vélez Vera, A. J., \& Villavicencio Briones, W. V. (2021). Causas de hemorragia postparto. RECIMUNDO, 5(1), 191-207. https://doi.org/10.26820/recimundo/5.(1).enero.2021.191-206

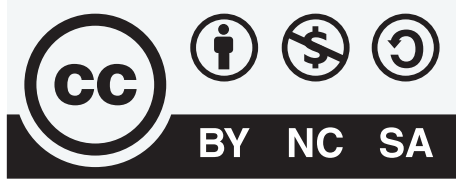

CREATIVE COMMONS RECONOCIMIENTO-NOCOMERCIAL-COMPARTIRIGUAL 4.0 . 